\title{
Minimal Ablation of the Tibial Stump Using Bony Landmarks Improved Stability and Synovial Coverage Following Double-Bundle Anterior Cruciate Ligament Reconstruction
}

\author{
Yuya Kodama, MD, PhD, Takayuki Furumatsu, MD, PhD, Tomohito Hino, MD, Yusuke Kamatsuki, MD, and \\ Toshifumi Ozaki, MD, PhD \\ Department of Orthopaedic Surgery, Okayama University Graduate School of Medicine, Dentistry and Pharmaceutical Sciences, Okayama, Japan
}

\begin{abstract}
Purpose: To evaluate the clinical effects of using anatomical bony landmarks (Parsons' knob and the medial intercondylar ridge) and minimal ablation of the tibial footprint to improve knee anterior instability and synovial graft coverage after double-bundle anterior cruciate ligament reconstruction.

Materials and Methods: We performed a retrospective comparison of outcomes between patients who underwent reconstruction with minimal ablation of the tibial footprint, using an anatomical tibial bony landmark technique, and those who underwent reconstruction with wide ablation of the tibial footprint. Differences between the two groups were evaluated using second-look arthroscopy, radiological assessment of the tunnel position, postoperative anterior knee joint laxity, and clinical outcomes.

Results: Use of the anatomical reference and minimal ablation of the tibial footprint resulted in a more anterior positioning of the tibial tunnel, with greater synovial coverage of the graft postoperatively $(\mathrm{p}=0.01$ ), and improved anterior stability of the knee on second-look arthroscopy. Both groups had comparable clinical outcomes.

Conclusions: Use of anatomical tibial bony landmarks that resulted in a more anteromedial tibial tunnel position improved anterior knee laxity, and minimal ablation improved synovial coverage of the graft; however, it did not significantly improve subjective and functional short-term outcomes.
\end{abstract}

Keywords: Knee, Anterior cruciate ligament, Reconstruction, Double-Bundle, Tibial bony landmark

\section{Introduction}

Several studies have sought the optimal reconstruction method to improve the mechanical stability of anterior cruciate ligament (ACL) grafts with regard to graft choice, number of bundles,

Received April 12, 2018; Revised (1st) July 12, 2018;

(2nd) July 26, 2018; Accepted August 9, 2018

Correspondence to: Takayuki Furumatsu, MD, $\mathrm{PhD}$

Department of Orthopaedic Surgery, Okayama University Graduate

School of Medicine, Dentistry and Pharmaceutical Sciences, 2-5-1

Shikatacho, Kitaku, Okayama 700-8558, Japan

Tel: +81-86-235-7273, Fax: +81-86-223-9727

E-mail: matino@md.okayama-u.ac.jp

This is an Open Access article distributed under the terms of the Creative Commons Attribution Non-Commercial License (http://creativecommons.org/licenses/by-nc/4.0/) which permits unrestricted non-commercial use, distribution, and reproduction in any medium, provided the original work is properly cited. preservation of the remnant, and tunnel positioning ${ }^{1-4)}$. Preservation of the remnant has been shown to provide several advantages, including better vascularization and synovial coverage of the graft $^{5,6)}$, preservation of the mechanoreceptors, and facilitation of mesenchymal stem cell migration ${ }^{7-9)}$. However, the clinical benefit of remnant preservation has not been clearly established, with recent meta-analyses reporting similar clinical outcomes and mechanical stability after ACL reconstruction performed with and without remnant preservation ${ }^{10,11)}$. Furthermore, although one study has reported on the importance of preserving a tibial stump as a minimum, if the remnant cannot be fully preserved ${ }^{12)}$, another study demonstrated that the tunnel position rather than remnant preservation is more important to reproduce the function of the original ACL after reconstruction ${ }^{13)}$.

Functional stability would further be enhanced after reconstruction by inducing good synovial coverage of the graft and by 
accurately placing aperture of the tibial tunnel within the tibial footprint of the ACL. We focused on two aspects of the reconstruction technique mainly to acquire good synovial coverage and to create a reproducible anatomical tibial tunnel: preservation of a tibial stump by the use of minimal ablation (MA) of the tibial footprint of the ACL ${ }^{12)}$ and the use of bony landmarks (Parsons' $\mathrm{knob}^{14)}$ and the medial intercondylar ridge ${ }^{15)}$ ) on the tibia to determine the position of the tibial tunnel. Our aim was to compare the outcomes of our double-bundle ACL reconstruction, using bony anatomical landmarks on the tibia performed with MA of tibial stump, to those of double-bundle reconstruction performed with wide ablation (WA) of the tibial stump of the ACL. We hypothesized that MA would improve synovial coverage of the graft and that placement of the tibial tunnel based on the tibial bony landmarks during ACL reconstruction would enhance anterior knee stability post-reconstruction.

\section{Materials and Methods}

This study was approved by our Institutional Review Board, and all patients provided informed consent prior to participation. Between January 2012 and March 2015, 83 consecutive patients underwent an outside-in double-bundle ACL reconstruction using hamstring autografts, performed by 2 surgeons. The medical records and intraoperative arthroscopic videos for these patients were reviewed to determine the procedure used: MA versus WA and tunnel positioning with or without reference to the bony landmarks. For procedures performed between January 2012 and March 2014, WA of the ACL stump was used, with creation of anteromedial (AM) and posterolateral (PL) tibial tunnels (WA group). Since April 2014, we modified our surgical technique on the tibial side of the reconstruction, using Parsons' knob and the medial intercondylar ridge to position the tibial tunnel and attempting to preserve as much of the ACL stump as possible (MA group), for following two reasons: first, we hoped the native ACL fibroblasts would be incorporated as part of the new composite graft to enhance synovial coverage of the graft; and second, preservation of the remaining ACL mechanoreceptors might have a beneficial effect on clinical outcomes ${ }^{12)}$. Among the 83 patients who underwent ACL reconstruction during the study period, 26 underwent the MA procedure and the remaining 57, the conventional WA procedure. From this latter group of 57, we selected 26 patients, matched on demographics to the 26 patients in the MA group.

\section{Evaluation of Outcomes}

The relevant characteristics of the study group are reported in Table 1, with no preoperative difference between the two groups identified. Outcomes were assessed based on measurement of the position of the AM and PL tibial tunnels on reconstructed computed tomography (CT) images, direct examination of the graft during second-look arthroscopy, quantification of anterior knee stability, and patient-reported clinical outcomes measured 2 years after reconstruction, including the Lysholm knee score, the International Knee Documentation Committee (IKDC) score, and the Knee Injury and Osteoarthritis Outcome Score (KOOS).

\section{Surgical Procedure}

A double-bundle, outside-in arthroscopic ACL reconstruction was performed in all patients, with the graft formed using the semitendinosus tendon (ST) and, if necessary, the gracilis tendon, as follows. A double bundle was constructed solely from the ST when the harvested ST was $>24 \mathrm{~cm}$, with the tendon cut transversely into two equal portions. When the harvested ST was $<24$ $\mathrm{cm}$, additional harvesting of the gracilis tendon was performed to obtain two equal portions (MA group, 3; WA group, 5). The harvested tendons were double-looped over an Endobutton fixation device (Smith \& Nephew, Andover, MA, USA), with the distal

Table 1. Demographic and Clinical Characteristics

\begin{tabular}{|c|c|c|c|}
\hline Characteristic & MA group & WA group & $\mathrm{p}$-value \\
\hline No. of patients & 26 & 26 & - \\
\hline Age (yr) & $26.5 \pm 9.2(16-41)$ & $23.7 \pm 5.2(16-33)$ & 0.60 \\
\hline Sex (male:female) & $12: 14$ & $13: 13$ & - \\
\hline Meniscus injury (rasping/meniscectomy/repair) & $14(7 / 1 / 6)$ & $13(5 / 2 / 6)$ & \\
\hline Body mass index $\left(\mathrm{kg} / \mathrm{m}^{2}\right)$ & $22.0 \pm 2.5(17.7-27.6)$ & $22.4 \pm 2.7(18.0-26.9)$ & 0.46 \\
\hline Interval from injury to ACL surgery (day) & $127.6 \pm 56.5(32-280)$ & $119.7 \pm 51.7(35-272)$ & 0.57 \\
\hline Interval from ACL surgery to second-look arthroscopy (day) & $419.7 \pm 61.9(253-417)$ & $411.4 \pm 133.8(273-452)$ & 0.23 \\
\hline
\end{tabular}

Values are presented as mean \pm standard deviation (range).

MA: minimal ablation, WA: wide ablation, ACL: anterior cruciate ligament. 
ends anchored using a Krackow suture, thus recreating the AM and PL bundles of the ACL. To prevent elongation of the grafts, a continuous 30 second loading with $70 \mathrm{~N}$ was applied twice to the graft ( $70 \mathrm{~N}, 1 \mathrm{~min})$, and then the same loading was repeatedly applied (70 N, $2 \mathrm{~min})^{16)}$. The femoral tunnel was created using an outside-in technique. The longitudinal linear resident's ridge ${ }^{17)}$ and the posterior cartilage, used as landmarks for the ACL femoral footprint, were identified. Two 2.4-mm guide pins were then inserted, separately, from the outside into the ACL footprint, posterior to the resident's ridge and just anterior to the articular margin, using an anterolateral-entry femoral aimer (Smith \& Nephew). A 5.5-mm to 6.5-mm tunnel was then created for the $\mathrm{AM}$ and the PL grafts by over-drilling of the guide pins. Two Endobutton-CLs (Smith \& Nephew) were connected to the end of each loop graft. The length of the CLs was matched to the length of the femoral tunnel so as to introduce sufficient graft materials (>13 mm) into the bone tunnels. The ACL remnant was resected with a shaver, preserving only the tibial stump.

\section{Creation of the Tibial Tunnels for the WA and MA Groups}

Using the conventional WA procedure, in the WA group, the anterior horn of the lateral meniscus (LM) was identified as a reference landmark ${ }^{18)}$, and the tunnel was created using WA within the ACL footprint. The AM tunnel was aligned with the anterior horn of LM, with the PL tunnel positioned in the posterior area within the footprint (Fig. 1). This WA approach does not preserve the tibial stump.

To preserve the tibial stump in the MA group, the AM tibial bony landmarks (Parsons' knob and the medial intercondylar ridge) were identified and ablation was limited to the medial side of the tibial footprint. The AM tunnel was created just lateral to the medial intercondylar ridge and just posterior to the Parsons' knob. The PL tunnel was created posterior to the AM tunnel and lateral to the medial intercondylar ridge, so as not to overlap with the AM tunnel (Fig. 1).

In all cases, tibial fixation of the graft was performed with the knee flexed at $20^{\circ}$, with an initial tension of $20 \mathrm{~N}$ applied to the PL bundle and $30 \mathrm{~N}$ to the AM bundle. The tension in each bundle was independently measured using a tensiometer ${ }^{19)}$.

\section{CT Image-Based Measurement}

CT images were obtained using an Asteion 4 Multislice CT System (Toshiba Medical Systems, Tochigi, Japan), at $120 \mathrm{kVp}$ and $150 \mathrm{~mA}$, with 1-mm slice thickness. CT reconstruction of the tibial condyles, in the axial plane, was completed using a threedimensional volume-rendering technique (AZE Virtual Place software; AZE Ltd., Tokyo, Japan). The position of the apertures of the tibial and femoral tunnels was obtained using a rectangular measurement grid. On the femoral side, the location of the insertion sites was described as a percentage of the distance both parallel and perpendicular to Blumensaat's line. On the tibial side, the location of the insertion sites was described as a percentage of the anteroposterior and mediolateral dimensions (Fig. 2).

\section{Second-Look Arthroscopic Examination}

Second-look arthroscopy was performed, with patients' consent, at approximately 1 year post-reconstruction for the removal of the two double-spike plates (Meira, Aichi, Japan), fixed with screws into the tibia, which were used for the tibial fixation of the ACL graft. These data were retrospectively collected from the
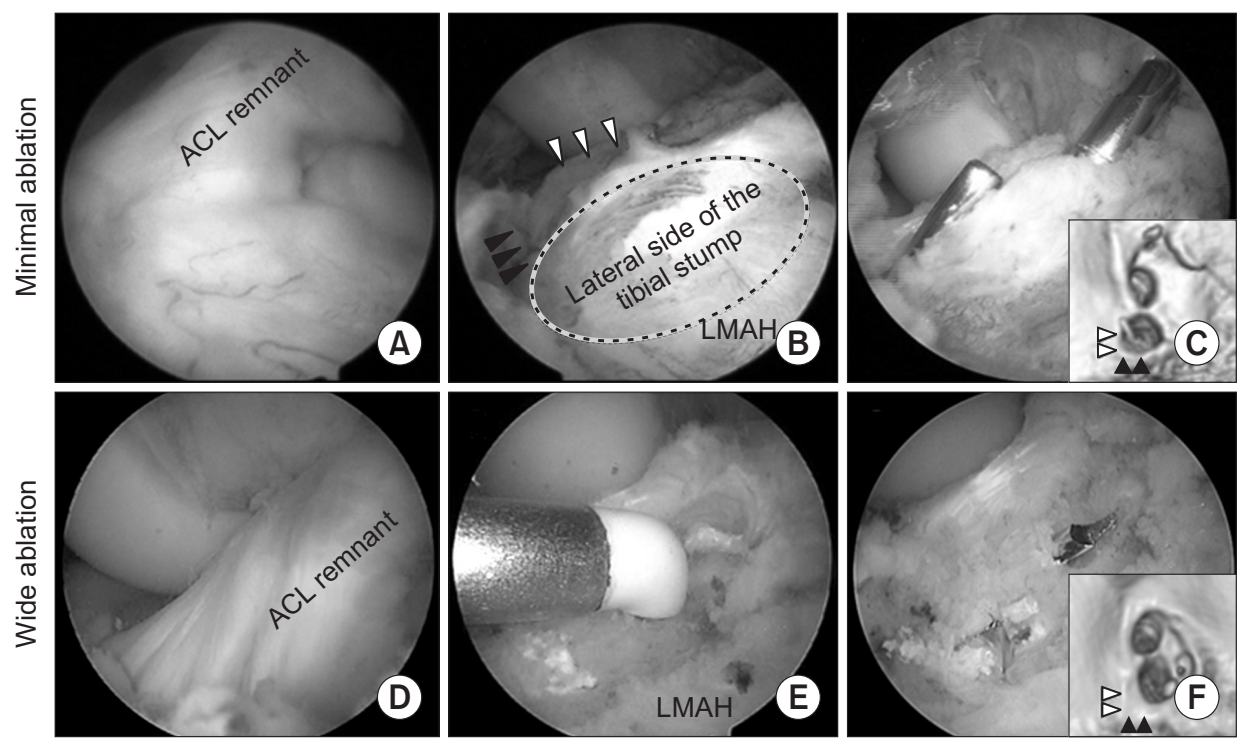

Fig. 1. Ablation range and tunnel creation within the anterior cruciate ligament (ACL) tibial footprint in the two groups. Tibial tunnel aperture of the left knee for the minimal ablation group (A-C), with ablation performed just medial to the footprint (B). (D-F) Wide ablation of the tibial stump, with no ACL stump retained. Black arrowheads: Parsons' knob, white arrowheads: medial intercondylar ridge, black circle: lateral side of the tibial stump, LMAH: lateral meniscus anterior horn. 

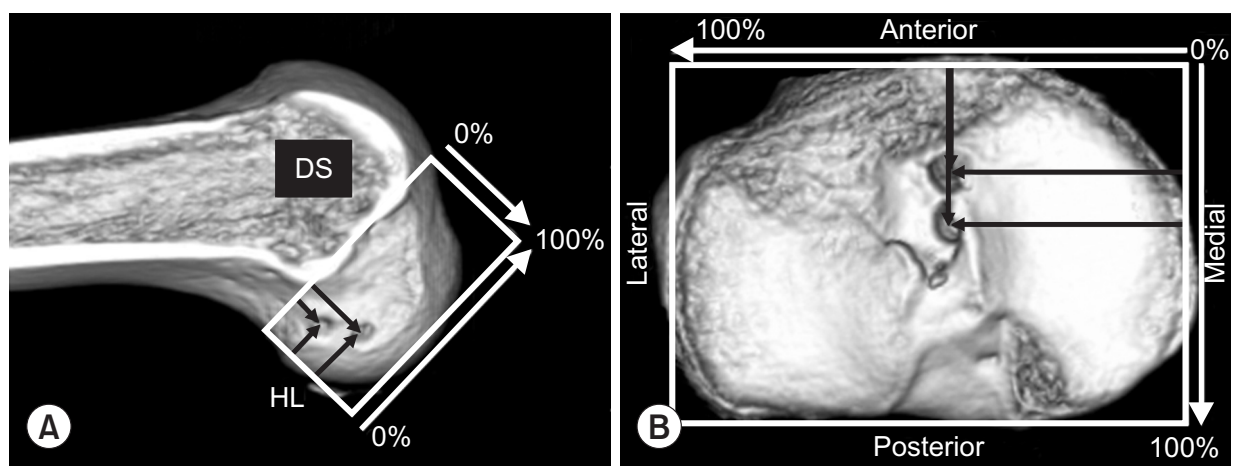

Fig. 2. Computed tomography image-based measurement of insertion sites in the femur and tibia. The total sagittal diameter of the lateral femoral condyle measured along Blumensaat's line. (B) The location of the insertion sites on the tibia was expressed as a percentage of the anterior-to-posterior and medial-to-lateral dimensions. DS: deep-to-shallow distance, HL: high-to-low distance.
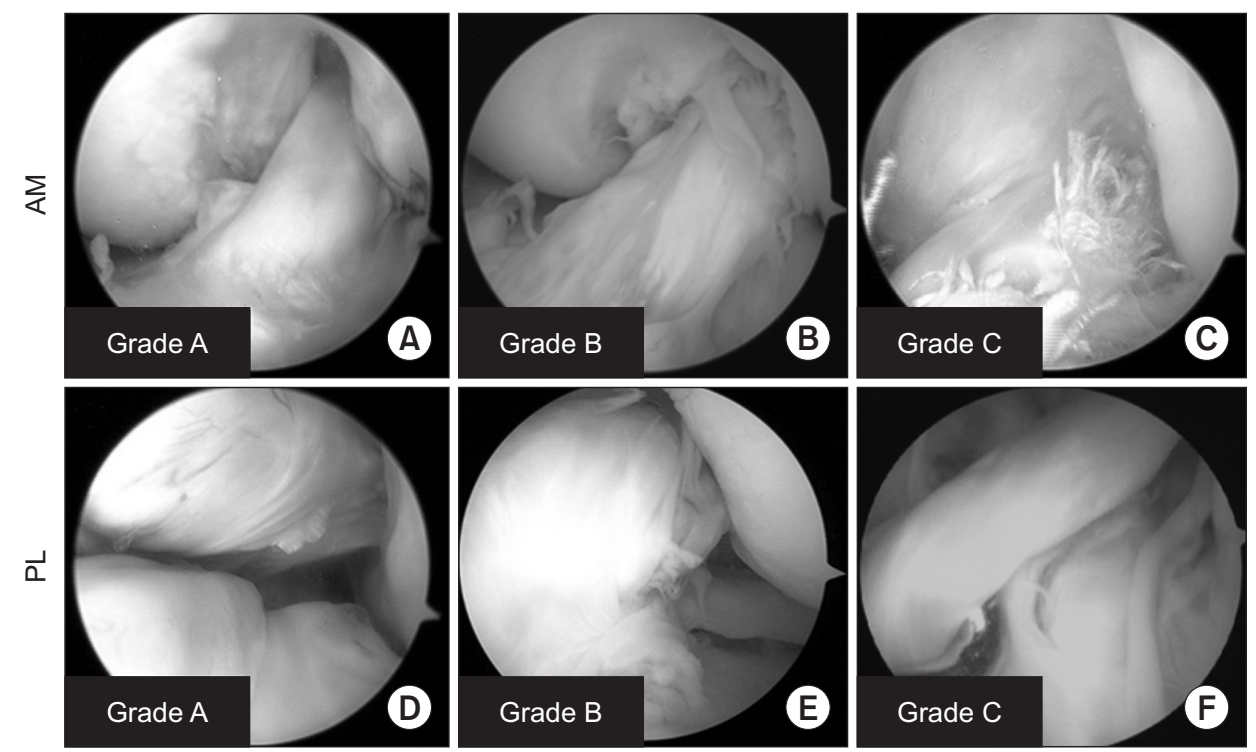

Fig. 3. Evaluation of synovial coverage. Arthroscopic grading of synovial coverage of the transplanted anteromedial (AM) (A$\mathrm{C})$ and posterolateral (PL) (D-F) bundle grafts. Grade A: synovium covers $>75 \%$ of the graft $(A, D)$, Grade B: coverage between $50 \%$ and $75 \%$ (B, E), Grade C: coverage $<50 \%$ (C, F).

records and confirmation from the review of arthroscopic videos. Arthroscopic evaluation was performed using the 3-point synovial coverage scale as reported by Kondo and Yasuda ${ }^{5}$, as follows: grade A, 3 points; grade B, 2 points, and grade $\mathrm{C}, 1$ point (Fig. 3 ).

\section{Evaluation of Clinical Outcomes}

Anterior knee laxity and rotational stability were measured under anesthesia by two experienced surgeons (TF and SM). Anterior tibial translation was assessed at the primary surgery and second-look evaluation using a KT-2000 arthrometer at maximum manual tension, with the knee in $20^{\circ}$ of flexion, as per a previously described procedure ${ }^{20)}$. Rotational stability was assessed using the manual pivot shift test, as previously described ${ }^{21)}$, at the time of the second-look arthroscopy. The pivot shift test was subjectively determined by the examiner based on the IKDC criteria: none (-), glide (+), clunk (++), or gross instability (+++). Functional outcomes were assessed 2 years after surgery using the Lysholm knee score, the IKDC form, and the KOOS, with scores obtained at each visit.

\section{Statistical Analyses}

Data were presented as means \pm standard deviations. Differences between the two groups were evaluated using the Mann-Whitney $U$-test, with a p-value $<0.05$ considered significant. Intra- and inter-observer repeatability in measurement of the position of the tibial tunnel was assessed by calculating the intraclass correlation coefficient (ICC), with the Kappa value used for grading of synovial coverage of the graft.

\section{Results}

\section{Tunnel Diameter and Position}

Comparison of the position of the tibial and femoral tunnels between the two groups is reported in Table 2. Measurement of the tunnel position was consistent, with ICC values of $0.899-0.912$ for intraobserver repeatability and 0.902-0.925 for interobserver repeatability. There was no significant difference in the position of the femoral tunnel between the MA and WA groups. However, there was a significant difference between the two groups in the 
Table 2. Comparison of the Position of the Tibial and Femoral Tunnels between the Two Groups

\begin{tabular}{lccc}
\hline Characteristic & MA group $(\mathrm{n}=26)$ WA group $(\mathrm{n}=26)$ & p-value \\
\hline Tibial tunnel & & & \\
AM bundle & & $35.2 \pm 4.5$ & $<0.01^{\mathrm{a})}$ \\
AP (\%) & $31.6 \pm 2.3$ & $46.8 \pm 1.7$ & $<0.01^{\mathrm{a})}$ \\
ML (\%) & $44.0 \pm 1.4$ & & \\
PL bundle & & $48.7 \pm 2.3$ & 0.10 \\
AP (\%) & $47.6 \pm 3.2$ & $46.1 \pm 1.5$ & $<0.01^{\mathrm{a})}$ \\
ML (\%) & $44.1 \pm 1.7$ & & \\
Femoral tunnel & & & \\
AM bundle & & $23.9 \pm 2.0$ & 0.71 \\
High-low & $23.7 \pm 1.7$ & $23.5 \pm 2.7$ & 0.50 \\
Deep-shallow & $22.9 \pm 1.9$ & & \\
PL bundle & & $50.1 \pm 2.4$ & 0.16 \\
High-low & $51.2 \pm 1.8$ & $31.5 \pm 2.4$ & 0.23 \\
Deep-shallow & $32.3 \pm 1.9$ & & \\
\hline
\end{tabular}

Values are presented as mean \pm standard deviation.

MA: minimal ablation, WA: wide ablation, AM: anteromedial, AP: anteroposterior, ML: mediolateral, PL: posterolateral.

${ }^{\text {a) }} \mathrm{p}<0.05$.

anteroposterior and mediolateral dimensions of the AM tibial tunnel $(31.6 \% \pm 2.3 \%$ and $44.0 \% \pm 1.4 \%$, respectively, for the MA group, and $35.2 \% \pm 4.5 \%$ and $46.8 \% \pm 1.7 \%$, respectively, for the WA group; $\mathrm{p}<0.01)$ and the mediolateral dimension of the PL tibial tunnel $(44.1 \% \pm 1.7 \%$ for the MA group and $46.1 \% \pm 1.5 \%$ for WA group; $\mathrm{p}<0.01)$.

\section{Synovial Coverage Evaluation}

Comparison of the arthroscopic evaluation between the two groups is reported in Table 3 . The determination of the grade of synovial coverage was consistent, with a Kappa value of 0.72 . Good synovial coverage (grade A) of the AM graft was identified in $85 \%$ of cases (22 knees) in the MA group and $54 \%$ of cases (14 knees) in the WA group, with poor coverage (grade C) identified in $8 \%$ of cases ( 2 knees) in the WA group. The synovial coverage scale of the AM bundle, based on Kondo's synovial coverage grade, was 2.8 \pm 0.4 in the MA group and $2.4 \pm 0.7$ in the WA group $(\mathrm{p}=0.03)$. No difference in the synovial coverage scale was identified between the two groups for the PL bundle ( $\mathrm{p}=0.51$ ).

\section{Clinical Outcomes}

A comparison of the clinical outcomes at the time of the second-look arthroscopy examination between the two groups is reported in Table 4 . The KT-2000 values were significantly
Table 3. Comparison of Arthroscopic Evaluation Results between the Two Groups

\begin{tabular}{lccc}
\hline \multicolumn{1}{c}{ Characteristic } & $\begin{array}{c}\text { MA group } \\
(\mathrm{n}=26)\end{array}$ & $\begin{array}{c}\text { WA group } \\
(\mathrm{n}=26)\end{array}$ & p-value \\
\hline AM synovial coverage, no. $(\%)$ & & & \\
Grade A & $22(85)$ & $14(54)$ & \\
Grade B & $4(15)$ & $10(38)$ & \\
Grade C & $0(0)$ & $2(8)$ & \\
Synovial coverage score, mean \pm SD & $2.8 \pm 0.4$ & $2.4 \pm 0.7$ & $0.03^{\text {a) }}$ \\
PL synovial coverage, no. $(\%)$ & & & \\
Grade A & $18(69)$ & $13(50)$ & \\
Grade B & $6(23)$ & $10(38)$ & \\
Grade C & $2(8)$ & $3(12)$ & \\
Synovial coverage score, mean \pm SD & $2.5 \pm 0.7$ & $2.3 \pm 0.7$ & 0.51 \\
\hline
\end{tabular}

Synovial coverage score was evaluated on a 3 -point scale: grade A, 3 points; grade $\mathrm{B}, 2$ points; and grade $\mathrm{C}, 1$ point.

MA: minimal ablation, WA: wide ablation, AM: anteromedial, SD: standard deviation, PL: posterolateral.

${ }^{\text {a) }} \mathrm{p}<0.05$.

Table 4. Comparison of the Clinical Outcome at the Final Follow-up between the Two Groups

\begin{tabular}{lccc}
\hline \multicolumn{1}{c}{ Characteristic } & $\begin{array}{c}\text { MA group } \\
(\mathrm{n}=26)\end{array}$ & $\begin{array}{c}\text { WA group } \\
(\mathrm{n}=26)\end{array}$ & p-value \\
\hline Lysholm knee score & $95.9 \pm 4.3$ & $95.7 \pm 6.6$ & 0.87 \\
IKDC evaluation & $83.7 \pm 9.7$ & $83.8 \pm 8.9$ & 0.97 \\
KOOS pain & $93.0 \pm 6.1$ & $92.7 \pm 5.6$ & 0.74 \\
Symptom & $92.4 \pm 7.9$ & $87.3 \pm 8.5$ & 0.06 \\
Activities of daily living & $98.1 \pm 4.8$ & $97.5 \pm 3.4$ & 0.54 \\
Sport/recreation & $85.5 \pm 12.7$ & $88.1 \pm 8.9$ & 0.59 \\
Quality of life & $86.7 \pm 11.1$ & $80.9 \pm 14.9$ & 0.19 \\
KT-2000 at second-look (mm) & $0.6 \pm 0.5$ & $1.4 \pm 0.8$ & $0.003^{\text {a) }}$ \\
Pivot shift test at second-look $(\%)$ & & & 0.84 \\
None (-) & $26(100)$ & $24(92)$ & \\
Glide (+) & 0 & $2(8)$ & \\
\hline
\end{tabular}

Values are presented as mean \pm standard deviation.

MA: minimal ablation, WA: wide ablation, IKDC: International Knee Documentation Committee, KOOS: Knee Injury and Osteoarthritis Outcome Score.

${ }^{a)} \mathrm{p}<0.05$.

lower for the MA group than for the WA group $(0.6 \pm 0.5 \mathrm{~mm}$ and $1.4 \pm 0.8 \mathrm{~mm}$, respectively, $\mathrm{p}<0.01)$. With regard to rotation stability, the pivot test was negative for 50 patients, with a positive "glide $(+)$ " assessed for 2 patients and with no differences between the two groups. Furthermore, there were no differences between the 
two groups in the Lysholm score, IKDC score, and KOOS.

\section{Discussion}

The most important findings of our study were that the use of anatomical tibial bony landmarks ${ }^{14,15)}$ (Parsons' knob and the medial intercondylar ridge) provided a more AM position of the AM tibial tunnel, improving anterior knee laxity. In addition, MA of the tibial stump was associated with better synovial coverage of the AM graft than WA.

Various landmarks for creating tibial tunnels in anatomically defined positions have been proposed to date ${ }^{22-24)}$. Until 2014, we used the anterior horn of the LM as a reference for tunnel positioning $^{18)}$, with this landmark being easily identified during arthroscopy. However, Ferretti et al. ${ }^{25)}$ stated that because of variations in the positional relationship between the anterior horn of the LM and the ACL attachment, this structure is unsuitable for use as a landmark to determine the positioning of the AM and PL tunnels. Tensho et al. ${ }^{15)}$ described that the tibial attachment of the ACL was located within a narrow area, surrounded by characteristic anatomic landmarks, based on their macroscopic and microscopic evaluations of the attachment sites of the ACL using 3-dimensional CT imaging. Based on this report, we thought that the use of two of these six bony landmarks would be sufficient to create a reproducible positioning of the tibial tunnel. In our study, positioning of the more AM tibial tunnel within the ACL footprint improved anterior stability of the knee after reconstruction. Moreover, MA, limited to the medial side of the tibial footprint, provided better visualization of the bony landmarks, which would improve the accuracy in the position of the AM tunnel. In addition to the tunnel position, we attempted to retain as much of the ACL stump as possible for two reasons. First, we considered that preservation of the remaining ACL mechanoreceptors might be beneficial to functional recovery, and second, we anticipated that the native ACL fibroblasts would be incorporated as part of the new composite graft.

The importance of preserving a tibial stump was reported by Schutte et al. ${ }^{12)}$ who demonstrated that the majority of mechanoreceptors are located close to the tibial insertion of the ACL. Although maintaining these mechanoreceptors could, theoretically, improve clinical outcomes at 2 years post-reconstruction, we did not identify a significant difference between the MA and WA groups. Although not reflected in our clinical results, good synovial coverage of the graft would further provide a potential source of nerve fibers, which could enhance the reinnervation of implanted grafts ${ }^{8)}$. We do demonstrate better synovial cover- age of the graft with the MA procedure. Based on research to date, it may be beneficial for ACL reconstruction to perform partial preservation on the tibial ACL stump and to achieve good synovial coverage, even if complete residual preservation is not achievable.

Using a tibial remnant preservation approach similar to ours, Lee et $\mathrm{al}^{26)}$ reported good proprioceptive and functional outcomes after ACL reconstruction. However, they positioned the tibial tunnel at the center of the normal ACL tibial remnant. Recent anatomical studies have provided evidence that positioning the tibial tunnel at the center of the ACL footprint may damage the anterior meniscal roots ${ }^{27-29)}$ and that an anterior tibial tunnel position improved anterior knee laxity post-reconstruction ${ }^{30)}$.

The limitations of our study need to be acknowledged. Foremost, the number of cases included in our analysis was limited $(n=52)$, and our analysis was retrospective in nature. Verification of our outcomes with large prospective studies is needed. Second, the follow-up period was within 2 years of ACL reconstruction. Therefore, we cannot speculate whether there will be differential effects between the two procedures on long-term outcomes of knee function. Third, the synovial tissue might contain some scar tissue, which would influence results. The amount of scar tissue in the synovial tissue should be objectively measured under microscope assessment. Lastly, although preservation of the remnant also preserves mechanoreceptors, we did not compare proprioceptive function between the two groups, due to the retrospective nature of the study.

\section{Conclusions}

Our findings suggest that a more AM tibial tunnel position would improve anterior knee laxity and MA would improve synovial coverage of the AM bundle of the graft.

\section{Conflict of Interest}

No potential conflict of interest relevant to this article was reported.

\section{Acknowledgments}

We are grateful to Dr. Shinichi Miyazawa and Masataka Fujii for their support. We would like to thank Editage (www.editage.jp) for English language editing. 


\section{References}

1. Duchman KR, Lynch TS, Spindler KP. Graft selection in anterior cruciate ligament surgery: who gets what and why? Clin Sports Med. 2017;36:25-33.

2. Adravanti P, Dini F, de Girolamo L, Cattani M, Rosa MA. Single-bundle versus double-bundle anterior cruciate ligament reconstruction: a prospective randomized controlled trial with 6-year follow-up. J Knee Surg. 2017;30:898-904.

3. Tibor L, Chan PH, Funahashi TT, Wyatt R, Maletis GB, Inacio MC. Surgical technique trends in primary ACL reconstruction from 2007 to 2014. J Bone Joint Surg Am. 2016;98: 1079-89.

4. Dhawan A, Gallo RA, Lynch SA. Anatomic tunnel placement in anterior cruciate ligament reconstruction. J Am Acad Orthop Surg. 2016;24:443-54.

5. Kondo E, Yasuda K. Second-look arthroscopic evaluations of anatomic double-bundle anterior cruciate ligament reconstruction: relation with postoperative knee stability. Arthroscopy. 2007;23:1198-209.

6. Ochi M, Adachi N, Deie M, Kanaya A. Anterior cruciate ligament augmentation procedure with a 1-incision technique: anteromedial bundle or posterolateral bundle reconstruction. Arthroscopy. 2006;22:463.

7. Bali K, Dhillon MS, Vasistha RK, Kakkar N, Chana R, Prabhakar S. Efficacy of immunohistological methods in detecting functionally viable mechanoreceptors in the remnant stumps of injured anterior cruciate ligaments and its clinical importance. Knee Surg Sports Traumatol Arthrosc. 2012;20: 75-80.

8. Dhillon MS, Bali K, Vasistha RK. Immunohistological evaluation of proprioceptive potential of the residual stump of injured anterior cruciate ligaments (ACL). Int Orthop. 2010; 34:737-41.

9. Fu W, Li Q, Tang X, Chen G, Zhang C, Li J. Mesenchymal stem cells reside in anterior cruciate ligament remnants in situ. Int Orthop. 2016;40:1523-30.

10. Ochi M, Abouheif MM, Kongcharoensombat W, Nakamae A, Adachi N, Deie M. Double bundle arthroscopic anterior cruciate ligament reconstruction with remnant preserving technique using a hamstring autograft. Sports Med Arthrosc Rehabil Ther Technol. 2011;3:30.

11. Ahn JH, Lee YS, Ko TS, Shin JY. Accuracy and reproducibility of the femoral tunnel with different viewing techniques in the ACL reconstruction. Orthopedics. 2016;39:e1085-91.

12. Schutte MJ, Dabezies EJ, Zimny ML, Happel LT. Neural anatomy of the human anterior cruciate ligament. J Bone Joint Surg Am. 1987;69:243-7.

13. Yasuda K, Kondo E, Ichiyama H, Tanabe Y, Tohyama H. Clinical evaluation of anatomic double-bundle anterior cruciate ligament reconstruction procedure using hamstring tendon grafts: comparisons among 3 different procedures. Arthroscopy. 2006;22:240-51.

14. Berg EE. Parsons' knob (tuberculum intercondylare tertium): a guide to tibial anterior cruciate ligament insertion. Clin Orthop Relat Res. 1993;(292):229-31.

15. Tensho K, Shimodaira H, Aoki T, Narita N, Kato H, Kakegawa A, Fukushima N, Moriizumi T, Fujii M, Fujinaga Y, Saito N. Bony landmarks of the anterior cruciate ligament tibial footprint: a detailed analysis comparing 3-dimensional computed tomography images to visual and histological evaluations. Am J Sports Med. 2014;42:1433-40.

16. Fujii M, Furumatsu T, Miyazawa $S$, Tanaka $T$, Inoue H, Kodama Y, Masuda K, Seno N, Ozaki T. Features of human autologous hamstring graft elongation after pre-tensioning in anterior cruciate ligament reconstruction. Int Orthop. 2016; 40:2553-8.

17. Shino K, Suzuki T, Iwahashi T, Mae T, Nakamura N, Nakata $\mathrm{K}$, Nakagawa S. The resident's ridge as an arthroscopic landmark for anatomical femoral tunnel drilling in ACL reconstruction. Knee Surg Sports Traumatol Arthrosc. 2010;18: 1164-8.

18. Zantop T, Wellmann M, Fu FH, Petersen W. Tunnel positioning of anteromedial and posterolateral bundles in anatomic anterior cruciate ligament reconstruction: anatomic and radiographic findings. Am J Sports Med. 2008;36:65-72.

19. Hamada M, Shino K, Horibe S, Mitsuoka T, Toritsuka Y, Nakamura N. Changes in cross-sectional area of hamstring anterior cruciate ligament grafts as a function of time following transplantation. Arthroscopy. 2005;21:917-22.

20. Daniel DM, Malcom LL, Losse G, Stone ML, Sachs R, Burks $\mathrm{R}$. Instrumented measurement of anterior laxity of the knee. J Bone Joint Surg Am. 1985;67:720-6.

21. Galway HR, MacIntosh DL. The lateral pivot shift: a symptom and sign of anterior cruciate ligament insufficiency. Clin Orthop Relat Res. 1980;(147):45-50.

22. Iriuchishima T, Ingham SJ, Tajima G, Horaguchi T, Saito A, Tokuhashi Y, Van Houten AH, Aerts MM, Fu FH. Evaluation of the tunnel placement in the anatomical double-bundle ACL reconstruction: a cadaver study. Knee Surg Sports Traumatol Arthrosc. 2010;18:1226-31.

23. Purnell ML, Larson AI, Clancy W. Anterior cruciate liga- 
ment insertions on the tibia and femur and their relationships to critical bony landmarks using high-resolution volume-rendering computed tomography. Am J Sports Med. 2008;36:2083-90.

24. Shimodaira H, Tensho K, Akaoka Y, Takanashi S, Kato H, Saito N. Remnant-preserving tibial tunnel positioning using anatomic landmarks in double-bundle anterior cruciate ligament reconstruction. Arthroscopy. 2016;32:1822-30.

25. Ferretti M, Doca D, Ingham SM, Cohen M, Fu FH. Bony and soft tissue landmarks of the ACL tibial insertion site: an anatomical study. Knee Surg Sports Traumatol Arthrosc. 2012;20:62-8.

26. Lee BI, Min KD, Choi HS, Kim JB, Kim ST. Arthroscopic anterior cruciate ligament reconstruction with the tibialremnant preserving technique using a hamstring graft. Arthroscopy. 2006;22:340.

27. Furumatsu T, Kodama Y, Maehara A, Miyazawa S, Fujii M,
Tanaka T, Inoue H, Ozaki T. The anterior cruciate ligamentlateral meniscus complex: a histological study. Connect Tissue Res. 2016;57:91-8.

28. Kodama Y, Furumatsu T, Miyazawa S, Fujii M, Tanaka T, Inoue $\mathrm{H}$, Ozaki T. Location of the tibial tunnel aperture affects extrusion of the lateral meniscus following reconstruction of the anterior cruciate ligament. J Orthop Res. 2017;35:162533.

29. LaPrade CM, Ellman MB, Rasmussen MT, James EW, Wijdicks CA, Engebretsen L, LaPrade RF. Anatomy of the anterior root attachments of the medial and lateral menisci: a quantitative analysis. Am J Sports Med. 2014;42:2386-92.

30. Bedi A, Maak T, Musahl V, Citak M, O’Loughlin PF, Choi D, Pearle AD. Effect of tibial tunnel position on stability of the knee after anterior cruciate ligament reconstruction: is the tibial tunnel position most important? Am J Sports Med. 2011;39:366-73. 